\title{
Crystal Fields in Dilute Rare-Earth Metals Obtained from Magnetization Measurements on Dilute Rare-Earth Alloys
}

\author{
Touborg, P.; Høg, J.
}

Published in:

Physical Review Letters

Link to article, DOI:

10.1103/PhysRevLett.33.775

Publication date:

1974

Document Version

Publisher's PDF, also known as Version of record

Link back to DTU Orbit

Citation (APA):

Touborg, P., \& Høg, J. (1974). Crystal Fields in Dilute Rare-Earth Metals Obtained from Magnetization Measurements on Dilute Rare-Earth Alloys. Physical Review Letters, 33(13), 775-778.

https://doi.org/10.1103/PhysRevLett.33.775

\section{General rights}

Copyright and moral rights for the publications made accessible in the public portal are retained by the authors and/or other copyright owners and it is a condition of accessing publications that users recognise and abide by the legal requirements associated with these rights.

- Users may download and print one copy of any publication from the public portal for the purpose of private study or research.

- You may not further distribute the material or use it for any profit-making activity or commercial gain

- You may freely distribute the URL identifying the publication in the public portal 


\title{
Crystal Fields in Dilute Rare-Earth Metals Obtained from Magnetization Measurements on Dilute Rare-Earth Alloys
}

\author{
P. Touborg and J. H $\phi \mathrm{g}$ \\ Department of Electrophysics, Technical University, DK-2800 Lyngby, Denmark
}

(Received 22 April 1974)

\begin{abstract}
Crystal field parameters of $\mathrm{Tb}, \mathrm{Dy}$, and $\mathrm{Er}$ in Sc, Y, and Lu are summarized. These parameters are obtained from magnetization measurements on dilute single crystals, and successfully checked by a number of different methods. The crystal field parameters vary unpredictably with the rare-earth solute. $B_{40}, B_{60}$, and $B_{66}$ are similar in Y and Lu. Crystal field parameters for the pure metals $\mathrm{Tb}$, $\mathrm{Dy}$, and $\mathrm{Er}$ are estimated from the crystal fields in $\mathrm{Y}$ and $\mathrm{Lu}$.
\end{abstract}

The rare-earth metals exhibit a fascinating variety of magnetic properties, which can be qualitatively understood as the consequence of crystal field, exchange, and magnetoelastic interactions. As a result of this complexity it is extremely dif ficult to separate individual terms. The crystal field plays a vital role in the magnetic ordering, in the spin-wave excitations, and in other magnetic properties of the rare-earth metals. ${ }^{1}$ It is therefore of great importance to know the crystal fields in the pure rare-earth metals. How ever, existing theories for the crystal field parameters, such as the point-charge model, are not expected to be of any validity, ${ }^{2}$ though the point-charge model gives good results for the metallic rare-earth pnictides. ${ }^{3}$ The crystal field parameters which have so far been determined experimentally ${ }^{1}$ have not shown satisfactory agreement among the results from different measurements. Recently the presence of highly anisotropic exchange interactions in the rare-earth metals has been demonstrated by the technique of inelastic neutron diffraction, ${ }^{4}$ and because of contributions from anisotropic exchange to the magnetic anisotropy, no reliable values of the crystal fields have been available yet. It is therefore understandable that the theoretical picture of the crystal fields in the rare-earth metals is also in a poor state. ${ }^{5}$

By diluting rare earths in nonmagnetic hosts, crystal-field-dominated systems can be created, in which an accurate determination of the crystal field is possible. An extensive project on dilute rare earths in nonmagnetic rare-earth-like host metals has been initiated, and in this Letter we wish to report crystal field parameters obtained for 0.1-1.0-at.\% alloys of $\mathrm{Tb}, \mathrm{Dy}$, and $\mathrm{Er}$ in the host metals Sc, Y, and Lu. These measurements have allowed-for the first time - a separate quantitative determination of the crystal field parame- ters in the pure rare-earth metals.

Sc, Y, and Lu are the only nonmagnetic elements which have rare-earth-like electronic structure and hcp crystal structures like the magnetic heavy rare-earth metals. $\mathrm{Y}$ and Lu bear very close resemblance to these, and the variations of the lattice parameters and the width of the $d$ conduction bands in $\mathrm{Tb}, \mathrm{Dy}$, and $\mathrm{Er}$ are bounded by $\mathrm{Y}$ and $\mathrm{Lu}$. Lu is a rare earth itself with a $5 d$ bandwidth approximately equal to those of $\mathrm{Tb}$, Dy, and Er. ${ }^{6}$ Determinations of the crystal fields for rare-earth impurities in $\mathrm{Y}$ and $\mathrm{Lu}$ are therefore expected to give good estimates of the crystal field parameters in the pure rareearth metals.

Crystal fields of dilute rare earths in cubic hosts have been determined earlier by spectroscopic $^{7}$ and by magnetization measurements. ${ }^{2}$ A determination of the axial anisotropy parameter from torque measurements on dilute rare earths in Gd has been attempted. ${ }^{89}$ However, the parameters obtained in this way are determined with low accuracy and do not agree with the parameters obtained in the present work. This is attributed to the severe difficulties in performing and interpreting such a torque experiment. ${ }^{9}$

In the present project the crystal fields in Sc, $\mathrm{Y}$, and $\mathrm{Lu}$ alloys were determined by fits to magnetization measurements. A recent paper ${ }^{10} \mathrm{de}-$ scribes the method used for Y:0.14-at.\% Er and Y:0.14-at.\% Dy. Because of the lower symmetry in the hcp hosts compared to the cubic systems, ${ }^{27}$ it was essential that the measurements were performed on single crystals. The samples were spheres cut from single-crystal ingots prepared by strain-anneal techniques. ${ }^{11}$ Magnetization measurements in the temperature range $1.3-300 \mathrm{~K}$ and in fields up to $6 \mathrm{~T}$ were performed in the $a, b$, and $c$ directions of the hcp structures. The mag- 
netic susceptibility of the host metal was measured separately and subtracted. The crystal field parameters were obtained by theoretical fits to the inverse initial susceptibility (measured by a high-sensitivity Faraday magnetometer ${ }^{10}$ ). Isofield and isothermal magnetization curves in the available temperature and field ranges (measured on the Faraday system and on a $5-\mathrm{Hz}$ vibratingsample magnetometer ${ }^{10}$ ) were used to check the reliability of the deduced parameters and to confirm the general validity of the Hamiltonian used. The inverse-initial-susceptibility curves show characteristic features for all Er and Dy alloys, allowing an unambiguous determination of the crystal field parameters. Figure 1 illustrates the accuracy of the fit for Sc:0.568-at.\% Dy. Ordering effects, etc. (Fig. 1), cause deviations at low temperatures and are responsible for the uncertainties on the crystal field parameters.

The $\mathrm{Tb}$ susceptibility curves contained too little structure to allow a unique determination of the parameters. For this case it was necessary to include results of additional measurements of the hexagonal anisotropy in the fitting procedure. ${ }^{12}$ These measurements were performed on a 179$\mathrm{Hz}$ vibrating-sample magnetometer, in which the components of the magnetic moment parallel and perpendicular to the external field, lying in the basal plane, were measured as a function of crystal rotation angle about the $c$ axis.

The Hamiltonian appropriate for the interpretation of these experiments is

$$
\mathfrak{H}=\mathfrak{H}_{\mathrm{cf}}+\mathfrak{H}_{\mathrm{Z}},
$$

where $\mathfrak{H}_{\mathrm{cf}}$ is the most general single-ion hexagonal anisotropy operator:

$$
\mathcal{H}_{\mathrm{cf}}=B_{20} \mathrm{O}_{20}+B_{40} \mathrm{O}_{40}+B_{60} \mathrm{O}_{60}+B_{66} \mathrm{O}_{66} \text {. }
$$

$\mathrm{O}_{l m}$ are the Stevens operators ${ }^{13}$ and $B_{l m}$ are crystal field parameters. $\mathcal{H}_{Z}$ is the effective Zeeman operator which includes the exchange interaction in the molecular-field approximation,

$$
\mathfrak{H}_{Z}=g_{J} \mu_{B} \vec{J} \cdot(\overrightarrow{\mathrm{H}}+\vec{\lambda} \cdot \overleftrightarrow{\mathrm{M}}) \text {. }
$$

$\vec{\lambda}$ is the molecular-field tensor containing two independent parameters, $\lambda_{\perp}$ and $\lambda_{\|}$.

During the fit, $\mathcal{H}_{\mathrm{cf}}$ was diagonalized in the subset of the lowest- $J$ multiplet, as contributions from the higher multiplets in the $L S$ ground-state term were found to be negligible. The four $B_{l m}$ 's, $\lambda_{\perp}, \lambda_{\|}$, and the atomic concentration $c$ were taken as fitting parameters. The deduced value of $c$ was always in agreement with the nominal concentration, within a few percent of the latter.
Because of the importance of knowing the crystal fields in the rare-earth metals it is preferable to check the parameters by other measurements. First we have studied the alloys $\mathrm{Y}: \mathrm{Tb}$, $\mathrm{Y}: \mathrm{Dy}$, and Y:Er for varying concentrations in the range $0.1-3.6$ at $\%{ }^{12}$ The magnetization measurements on these systems were excellently described by concentration-independent crystal field parameters. Secondly we have performed measurements of the basal-plane anisotropy on almost all alloys. ${ }^{12}$ For the Er and Dy alloys, where these measurements are not included in the fitting procedure, they have served as a separate check of the $B_{66}$ parameter. Magnetization measurements on $\mathrm{Y}: \mathrm{Tb}, \mathrm{Y}: \mathrm{Dy}$, and $\mathrm{Y}: \mathrm{Er}$ in fields up to $370 \mathrm{kOe}$, where the Zeeman energies are comparable to the crystal field energies, are described well by the crystal field parameters. ${ }^{14}$ This includes characteristic features due to mixing and crossing of crystal field levels. Finally three expected transitions from the ground state to excited crystal field levels in an Y:Er single crystal have been observed with correct energies and intensities in an inelastic-neutron-scattering experiment. ${ }^{15}$

In Fig. 2 the crystal field parameters divided by the Stevens factors ${ }^{13}$ are plotted as functions of the $c / a$ ratio of the host metal. For comparison curves calculated in the point-charge model ${ }^{16}$ are also shown. The deviation of these from the experimental data demonstrates the failure of the point-charge model also for these systems. The point-charge model even predicts the wrong sign

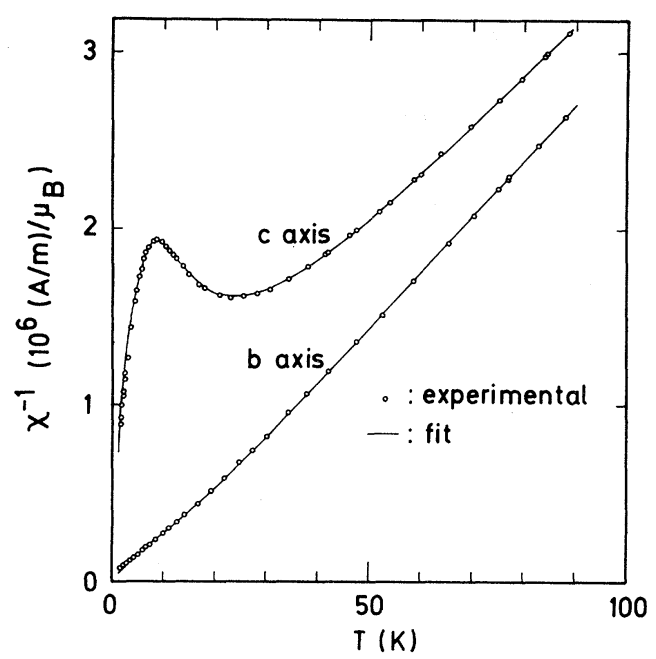

FIG. 1. Inverse susceptibility of Sc:0.568-at\% Dy measured at $B=0.170 \mathrm{~T}$. The susceptibility is given per Dy atom. 

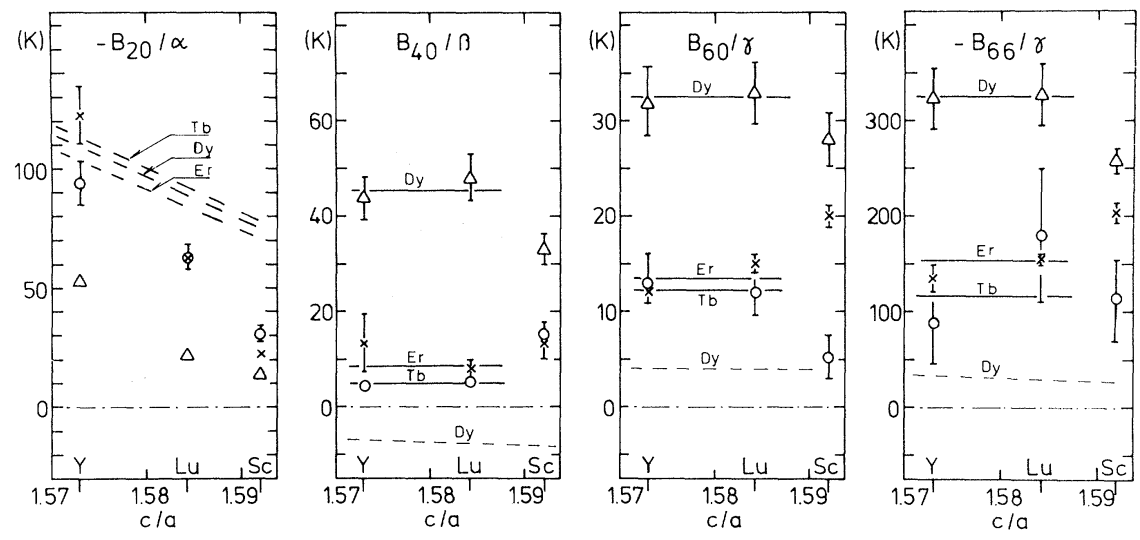

FIG. 2. Crystal field parameters divided by Stevens factors versus $c / a$ ratio for Tb (circles), Dy (triangles), and Er (crosses). Errors are not plotted when they fall within the simbol. The full lines represents the average of the $\mathrm{Y}$ and Lu results. The dashed lines are calculated from the point-charge model (Ref. 16). Except for $B_{20} / \alpha$, the point-charge parameters for $\mathrm{Tb}$ and $\mathrm{Er}$ are insignificantly different from those for Dy and are therefore not plotted.

of $B_{40} / \beta$. The $B_{l m}$ 's divided by the corresponding Stevens factors have, for given $l$ and $m$, the same sign for all alloys, and the ratio $-B_{66} / B_{60}$ for any given alloy is approximately 10 . A substantial variation in magnitude for different rare-earth impurities in the same host is observed, with $\mathrm{Tb}$ and $\mathrm{Er}$ being most alike. The energy $V_{\text {cf }}$ of a $4 f$ electron in a crystalline electric field with hcp symmetry can be written as

$$
\begin{aligned}
V_{\mathrm{cf}}=4(\pi / 5)^{1 / 2} V_{20}(r) Y_{20}(\theta, \varphi)+(16 / 3) & \pi^{1 / 2} V_{40}(r) Y_{40}(\theta, \varphi)+32(\pi / 13)^{1 / 2} V_{60}(r) Y_{60}(\theta, \varphi) \\
& +32(\pi / 3003)^{1 / 2} V_{66}(r)\left[Y_{66}(\theta, \varphi)+\boldsymbol{Y}_{6,-6}(\theta, \varphi)\right] .
\end{aligned}
$$

$V_{l m}(\gamma)$ describes the radial part of the electric field component with the angular dependence $Y_{l m}(\theta, \varphi)$. The connection to the Hamiltonian (2) is given by $B_{l m} / \alpha_{l}=\left\langle V_{l m}\left(v_{4 f}\right)\right\rangle .^{13}$ The $V_{l m}$ 's may contain contributions from charges both within and outside the impurity ion. The external contribution has the form $A_{l m} r^{l}$ and is not expected to depend very much on the nature of the impurity ion, which however may greatly influence the internal contribution. It may therefore be concluded that the charge residing on the magnetic ion makes a significant contribution to the crystal field which it experiences.

In order to obtain estimates of the crystal field parameters for the pure rare-earth metals, we have shown in Fig. 2 the averages of the values for $B_{40} / \beta, B_{60} / \gamma$, and $B_{66} / \gamma$ measured in the hosts $\mathrm{Y}$ and $\mathrm{Lu}$. These values are, within the experimental error, equal for $\mathrm{Y}$ and $\mathrm{Lu} . B_{20} / \alpha$ is seen to vary considerably with the $c / a$ ratio, which is close to the ideal hcp value $\left(\frac{8}{3}\right)^{1 / 2}$. The dependence of $B_{20} / \alpha$ on $c / a$ is of the same order of magnitude as estimated from high-temperature paramagnetic measurements on the pure rareearth metals. ${ }^{17}$ The deviations in $B_{40} / \beta, B_{60} / \gamma$, and $B_{66} / \gamma$ observed for Sc probably reflect the difference between this metal and $\mathrm{Lu}$ and $\mathrm{Y}$ with respect to atomic volume and to the width of the $d$ conduction band. ${ }^{6}$ Crystal field parameters for the pure rare-earth metals obtained from the appropriate $\mathrm{Y}$ and $\mathrm{Lu}$ values by interpolation for $B_{20}$ and by taking the average values for $B_{40}, B_{60}$, and $\boldsymbol{B}_{66}$ are given in Table I.

In conclusion the measurements of crystal fields of rare-earth metals diluted in the hosts $\mathrm{Y}$ and Lu have allowed a determination of the crystal field parameters in the pure rare-earth metals. The crystal field parameters seem to vary unsystematically with the atomic number of the rare earth, contrary to what is expected in simple theories. This demonstrates the necessity of a first-principles calculation based on electron band structures.

TABLE I. Estimated crystal field parameters for the pure rare-earth metals.

\begin{tabular}{lrcrr}
\hline & $\begin{array}{c}B_{20} \\
\left(10^{-2} \mathrm{~K}\right)\end{array}$ & $\begin{array}{c}B_{40} \\
\left(10^{-4} \mathrm{~K}\right)\end{array}$ & $\begin{array}{c}B_{60} \\
\left(10^{-6} \mathrm{~K}\right)\end{array}$ & $\begin{array}{c}B_{66} \\
\left(10^{-6} \mathrm{~K}\right)\end{array}$ \\
\hline $\mathrm{Tb}$ & $73 \pm 6$ & $6 \pm 1$ & $-13 \pm 3$ & $128_{-28}^{+112}$ \\
$\mathrm{Dy}$ & $32 \pm 2$ & $-27 \pm 3$ & $34 \pm 3$ & $\begin{array}{c}336_{ \pm} \pm 31 \\
317_{-0}^{+83}\end{array}$ \\
$\mathrm{Er}$ & $-32 \pm 3$ & $4_{-1}^{+5}$ & $28 \pm 6$ & \\
\hline
\end{tabular}


The authors are grateful to Professor V. Frank and Professor A. R. Mackintosh for valuable suggestions during the course of the research.

$\mathrm{K}$. Pedersen assisted in the preparation of singlecrystal samples.

${ }^{1}$ For a review, see Magnetic Properties of Rare Earth Metals, edited by R. J. Elliott, (Plenum, New York, 1972).

${ }^{2} \mathrm{G}$. Williams and L. L. Hirst, Phys. Rev. 185, 407 (1969).

${ }^{3}$ K. C. Turberfield, L. Passell, R. Birgeneau, and E. Bucher, Phys. Rev. Lett. 25, 752 (1970), and Phys. Rev. B $\underline{4}, 718$ (1971).

${ }^{4} \mathrm{~J}$. Jensen, J. G. Houmann, and H. Bjerrum Mфller, to be published; R. M. Nicklow, N. Wakabayashi, M. K. Wilkinson, and R. E. Reed, Phys. Rev. Lett. 27, 334 (1971).

${ }^{5}$ For a review, see A. J. Freeman, in Ref. 1. ${ }^{6} \mathrm{O}$. Jepsen and O. K. Andersen (private communication) quote the following values for the $d$-conductionband widths in units of rydbergs: Sc, 0.28; Y, 0.48; $\mathrm{Tb}, 0.59$; and $\mathrm{Lu}, 0.61$ (obtained by the standard potential construction using superposition of atomic change densities and full Slater exchange).

${ }^{7}$ D. Davidov, R. Orbach, C. Rettori, L. J. Tao, and E. P. Chock, Phys. Rev. Lett. 28, 490 (1972); R. A. Devine, J. M. Moreti, J. Ortelli, D. Shaltiel, W. Zingg, and M. Peter, Solid State Commun. 10, 575 (1972); C. Rettori, D. Davidov, R. Orbach, and E. P. Chock, Phys Rev. B 7, 1 (1973), and references therein.

${ }^{8}$ S. Chikazumi, K. Tajima, and K. Toyama, J. Phys. (Paris), Colloq. 32, C1-180 (1971).

${ }^{9}$ T. Asada, J. Phys. Soc. Jpn. 35, 85 (1973) .

${ }^{10} \mathrm{~J} . \mathrm{H} \not \mathrm{g}$ and P. Touborg, Phys. Rev. B $\underline{9}, 2920$ (1974).

${ }^{11}$ K. A. McEwen and P. Touborg, J. Phys. F: Metal Phys。․ 1903 (1973).

${ }^{12} \mathrm{~J} . \overline{\mathrm{H}} \not \mathrm{g}$ and P. Touborg, Phys. Rev. B $\underline{9}, 2920$ (1974).

${ }^{13} \mathrm{~A}$. Abragam and B. Bleaney, Electron Faramagnetic Resonance of Transition Ions (Clarendon Press, Oxford, England 1970).

${ }^{14}$ P. Touborg, J. Høg, G. J. Cock and L. W. Roeland, Phys。 Rev. B (to be published).

${ }^{15}$ O. Rathmann, J. Als-Nielsen, P. Bak, J. Høg and P. Touborg, Phys. Rev. B (to be published).

${ }^{16} \mathrm{~T}$. Kasuya, in Magnetism, edited by G. T. Rado and H. Suhl (Academic, New York, 1966), Vol. IIB.

${ }^{17} \mathrm{R}$. Aleonard, P. Boutron, and D. Bloch, J. Phys. Chem. Solids $\underline{30}, 2277$ (1969); P. Touborg, S. Balling, and K. M. Ravn, to be published.

\title{
Magnetic Properties of Ho-Co and Ho-Fe Amorphous Films
}

\author{
Neil Heiman and Kenneth Lee \\ IBM Research Laboratory, San Jose, Califormia 95193
}

(Received 6 May 1974)

\begin{abstract}
Polar-Faraday-rotation and magnetization measurements on evaporated amorphous films of Ho-Co and Ho-Fe show that the principle mechanism responsible for differences in the $T_{C}$ of the crystalline and amorphous states is the altered electronic configuration of the transition metal, rather than local anisotropy. Additionally we found that the films possess uniaxial anisotropy.
\end{abstract}

That the technologically important ${ }^{1.2}$ amorphous rare-earth (RE)-transition-metal (TM) alloys can be prepared ${ }^{1,3,4}$ without the need to include "glass-former" atoms or to maintain the samples at cryogenic temperatures presents an opportunity to understand the fundamentals of amorphous magnetism. Published experimental data on the magnetic properties of amorphous RE$\mathrm{TM}$ alloys are confined mainly to $\mathrm{TbFe}_{2}{ }^{4,5}$ and $\mathrm{GdCO}_{2}{ }^{1{ }^{18,7}}$ There is considerable interest in the fact that the Curie temperature $\left(T_{\mathrm{C}}\right)$ for $\mathrm{TbFe}_{2}$ is markedly lower in the amorphous state than in the crystalline state, whereas $T_{\mathrm{C}}$ of $\mathrm{GdCo}_{2}$ is considerably higher in the amorphous state than in the crystalline state. Harris, Plischke, and Zuckermann $^{8}$ attribute these differences to the random orientation of the local anisotropy field, in that $\mathrm{Gd}$ is an $S$-state atom whereas $\mathrm{Tb}$ is highly anisotropic. Tao et al., ${ }^{6.7}$ while not ruling out local-anisotropy effects altogether, attribute the differences to the fact that reduced density inhibits charge transfer from the RE to the $d$ orbitals of the TM. Since Fe, unlike Co, has unfilled majority as well as minority spin states, they indicate that reduced charge transfer may produce opposite results in $\mathrm{TbFe}_{2}$ and $\mathrm{GdCo}_{2}$. Unfortunately the data on amorphous $\mathrm{TbFe}_{2}$ and $\mathrm{GdCO}_{2}$ are not sufficient to resolve the question of the relative importance of the two mechanisms.

Additionally some existing data seem to argue against either mechanism. For example, a recent Letter by Sarkar et al. ${ }^{9}$ reports Mössbauer- 